\title{
A New Light on Photosystem II Maintenance in Oxygenic Photosynthesis
}

\author{
Jun Liu ${ }^{1,2 *}$, Yan Lü, Wei Hua ${ }^{1 *}$ and Robert L. Last ${ }^{2,4}$ \\ 'Department of Functional Genomics and Molecular Biology, Key Laboratory of Biology and Genetic Improvement of Oil \\ Crops, Ministry of Agriculture and Rural Affairs, Oil Crops Research Institute of the Chinese Academy of Agricultural \\ Sciences, Wuhan, China, ${ }^{2}$ Department of Biochemistry and Molecular Biology, Michigan State University, East Lansing, MI, \\ United States, ${ }^{3}$ Department of Biological Sciences, Western Michigan University, Kalamazoo, MI, United States, ${ }^{4}$ Department \\ of Plant Biology, Michigan State University, East Lansing, MI, United States
}

Life on earth is sustained by oxygenic photosynthesis, a process that converts solar energy, carbon dioxide, and water into chemical energy and biomass. Sunlight is essential for growth and productivity of photosynthetic organisms. However, exposure to an excessive amount of light adversely affects fitness due to photooxidative damage to the photosynthetic machinery, primarily to the reaction center of the oxygen-evolving

OPEN ACCESS

Edited by:

Cornelia Spetea,

University of Gothenburg, Sweden

Reviewed by:

Yoshitaka Nishiyama,

Saitama University, Japan

Peter J. Gollan,

University of Turku, Finland

${ }^{*}$ Correspondence:

Jun Liu

liujunocr@caas.cn

Wei Hua

huawei@oilcrops.cn

Specialty section: This article was submitted to

Plant Physiology,

a section of the journal

Frontiers in Plant Science

Received: 19 April 2019

Accepted: 11 July 2019

Published: 31 July 2019

Citation:

Liu J, Lu Y, Hua W and Last RL (2019) A New Light on

Photosystem II Maintenance in

Oxygenic Photosynthesis.

Front. Plant Sci. 10:975.

doi: 10.3389/fp/s.2019.00975 photosystem II (PSII). Photosynthetic organisms have evolved diverse photoprotective and adaptive strategies to avoid, alleviate, and repair PSIl damage caused by highirradiance or fluctuating light. Rapid and harmless dissipation of excess absorbed light within antenna as heat, which is measured by chlorophyll fluorescence as non-photochemical quenching (NPQ), constitutes one of the most efficient protective strategies. In parallel, an elaborate repair system represents another efficient strategy to maintain PSII reaction centers in active states. This article reviews both the reaction center-based strategy for robust repair of photodamaged PSII and the antenna-based strategy for swift control of PSIl light-harvesting (NPQ). We discuss evolutionarily and mechanistically diverse strategies used by photosynthetic organisms to maintain PSIl function for growth and productivity under static high-irradiance light or fluctuating light environments. Knowledge of mechanisms underlying PSII maintenance would facilitate bioengineering photosynthesis to enhance agricultural productivity and sustainability to feed a growing world population amidst climate change.

Keywords: photosystem II, photosynthesis, non-photochemical quenching, repair, fluctuating light

\section{INTRODUCTION}

Cyanobacteria, algae, and plants convert sunlight into chemical energy through photosynthesis to provide oxygen and food building blocks that are essential for most life forms on earth. Photosynthesis starts with capture of light by light-harvesting antenna, which drives photosynthetic electron flow through photosynthetic machinery comprising several large protein complexes embedded in the thylakoid membranes of prokaryotic cyanobacteria and eukaryotic chloroplasts. Oxygen-evolving photosystem II (PSII) is a highly conserved multi-subunit pigment-containing membrane complex 
that functions as a light-driven water:plastoquinone oxidoreductase during photosynthetic electron transport (reviewed in Kern and Renger, 2007; Koochak et al., 2019). The electrons extracted from water are converted and stored into organic molecules. Counterintuitively, PSII is extremely vulnerable to light irradiance, which causes photodamage to PSII reaction centers (reviewed in Townsend et al., 2018; Leister, 2019). The damage is exacerbated if light energy exceeds what can be utilized for carbon fixation, particularly when photosynthetic organisms are subjected to environmental stresses, such as high light, extreme temperature, drought and nutrient depletion, or combined stresses (Ghotbi-Ravandi et al., 2014; reviewed in Murata et al., 2007; Sainz et al., 2010; Salomon et al., 2013; Strzepek et al., 2019; Wilson and Ruban, 2019). The excess light energy also leads to massive generation of reactive oxygen species (ROS) photoproducts, which damage PSII or suppress the repair of damaged PSII (Mishra and Ghanotakis, 1994; Miyao et al., 1995; Okada et al., 1996; Nishiyama et al., 2001; Kale et al., 2017; reviewed in Pinnola and Bassi, 2018). Paradoxically, ROS also act as critical signal molecules to mediate photoacclimation response (Alboresi et al., 2011; reviewed in Wagner et al., 2004; Dogra et al., 2018).

Photoinhibition occurs when PSII suffers from excess lightinduced damage or PSII photochemistry is downregulated, resulting in decreased photosynthetic performance and reduced growth and productivity (Kapri-Pardes et al., 2007; Chen et al., 2019; reviewed in Takahashi and Badger, 2011; Wittenberg et al., 2014; Ting and Owens, 2016; Li et al., 2018). Photosynthetic organisms evolved a suite of photoprotective and adaptive mechanisms to prevent or recover from the deleterious effects of photoinhibitory light. These include fast regulatory mechanisms, for instance, movement of chloroplasts away from high-light intensity, reduction of antenna size, induction of alternative electron transport pathways, and slow regulatory mechanisms, such as operation of both enzymatic and non-enzymatic ROS scavenging systems, and triggering systemic acquired acclimation (reviewed in Jarillo et al., 2001; Frigerio et al., 2007; Okegawa et al., 2010; Erickson et al., 2015). Non-photochemical quenching (NPQ) represents one of the fast regulatory mechanisms that is immediately activated and rapidly inducible upon excess solar energy. It protects against excess absorbed sunlight within the PSII antenna by converting photons into dissipative heat (Niyogi et al., 1998; reviewed in Wobbe et al., 2016). In addition, certain organism-specific protein factors evolved to maintain maximal PSII activity under photoinhibitory light conditions (Chen et al., 2018). The land plant-specific thylakoid membrane proteins MPH1 (MAINTENANCE OF PSII UNDER HIGH LIGHT 1) and HHL1 (HYPERSENSITIVE TO HIGH LIGHT 1) evolved to protect PSII against high-light illumination following the transition from aquatic habitats to terrestrial environments (Jin et al., 2014; Liu and Last, 2015a,b). Despite these multi-faceted photoprotective mechanisms, light-induced damage to PSII still occurs. Photosynthetic organisms employ an efficient repair system to replace damaged subunits within PSII reaction centers and restore PSII function (reviewed in Li et al., 2018). A suite of auxiliary proteins, including kinases, phosphatase(s), proteases, and repair/assembly factors have been documented to promote the repair of damaged PSII core subunits (reviewed in
Nickelsen and Rengstl, 2013; Järvi et al., 2015). These auxiliary proteins could also cooperate with each other to facilitate the repair process. For instance, Arabidopsis thaliana (a flowering plant model species) LQY1 (LOW QUANTUM YIELD OF PHOTOSYSTEM II 1) protein-interacting with HHL1-regulates repair of damaged core complexes to sustain high PSII efficiency upon exposure to excessive light ( $\mathrm{Lu}, 2011$; Lu et al., 2011; Jin et al., 2014). Another example is the recent finding that OHP1 (ONE-HELIX PROTEIN1), OHP2, and HCF244 (HIGH CHLOROPHYLL FLUORESCENCE244) form a transient functional heterotrimeric complex assisting in assembly and/or repair of PSII (Hey and Grimm, 2018; Myouga et al., 2018; Li et al., 2019). These repair and NPQ systems may become especially important and could operate in parallel or synergistically to maintain optimal PSII efficiency under fluctuating light environments because photosynthetic organisms live in-and adapt to-their natural growth conditions where light fluctuates rapidly and unpredictably. This review focuses on antenna- and reaction center-based strategies that coexist in oxygenic organisms to minimize the production of the photosynthetic byproducts ROS, thereby safeguarding PSII under changes in light conditions.

\section{PREVENTION: REGULATION OF LIGHT CAPTURE AS A PHOTOPROTECTIVE MECHANISM ACROSS PHOTOSYNTHETIC ORGANISMS}

\section{Non-photochemical Quenching Regulation of Light-Harvesting Efficiency}

Photosynthesis is initiated by the capture and trapping of solar energy by light-harvesting systems in thylakoid membranes of cyanobacteria or chloroplasts. However, absorbed light that exceeds what can be used by photosynthesis causes light-induced damage, primarily to PSII. Therefore, maintenance of optimal photosynthetic performance requires efficient regulation of light harvesting for photoprotection. NPQ safely dissipates excess light energy within the PSII antenna system and is found ubiquitously across oxygenic photosynthetic organisms (reviewed in Niyogi and Truong, 2013).

NPQ responds rapidly and prevents ROS formation during photosynthesis (Figure 1). It is a protective strategy for photosynthetic machinery to acclimatize to excess light conditions. NPQ consists of a variety of processes, such as redistribution of antenna between PSII and PSI to balance electron transport (qT type of NPQ, or state transition) (Bellafiore et al., 2005; reviewed in Erickson et al., 2015), deepoxidation of violaxanthin into zeaxanthin in the xanthophyll cycle and global structural reorganization of PSII-LHCII complexes (Niyogi et al., 1998; Ruban et al., 2007; Park et al., 2019). The most prominent and fastest component is zeaxanthin-facilitated energy-dependent quenching ( $\mathrm{qE}$ type quenching or feedback de-excitation) (Li et al., 2000; Tian et al., 2019). Because it operates on a time scale of seconds to minutes, rapid and reversible $\mathrm{qE}$ is often referred to as flexible thermal dissipation (Demmig-Adams et al., 2006; 


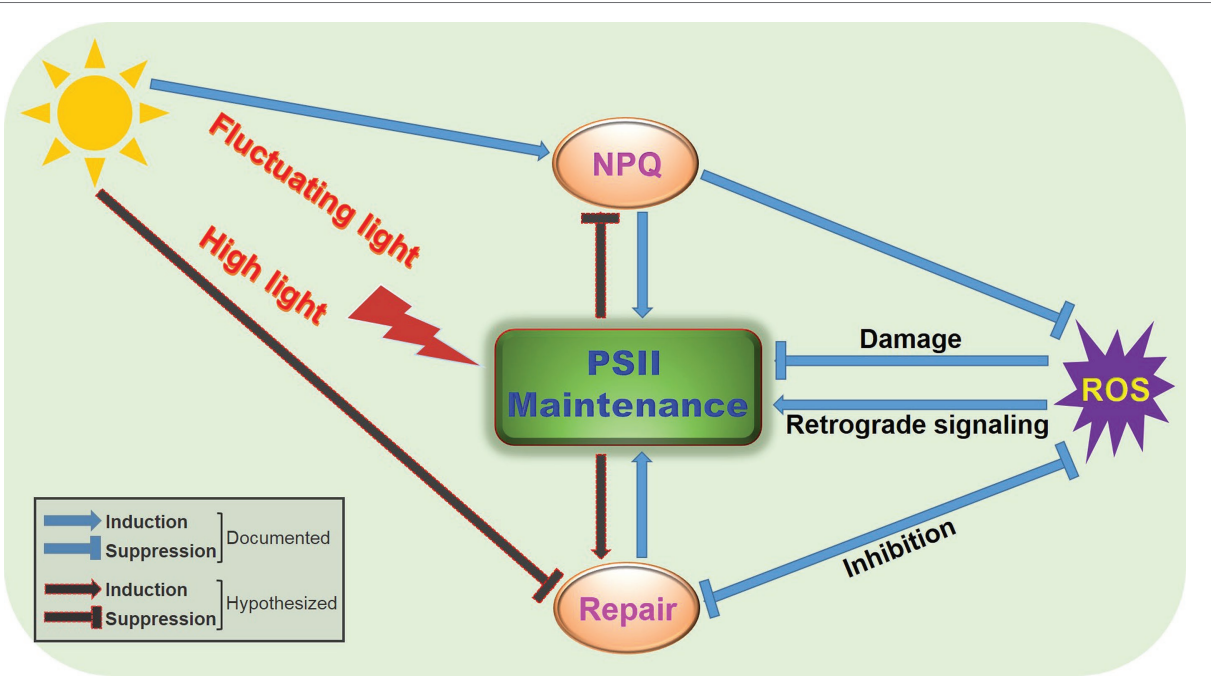

FIGURE 1 | A proposed simplified model illustrating regulation of PSIl function by NPQ and repair under fluctuating light environments or high-light irradiance. Fluctuating light or high light can cause damage to PSII and downregulation of PSII photochemistry, with concomitant generation of ROS. To maintain normal PSII function, photosynthetic organisms deploy the antenna-based strategy, NPQ, and the reaction center-based strategy, PSIl repair, to efficiently regulate light utilization and energy transfer. ROS act on PSII through damage, inhibition of repair, or retrograde signaling, whose production can be decreased by NPQ or repair. These intricate interplays between NPQ and repair can optimize PSIl performance and facilitate acclimation of photosynthetic groups to fluctuating light environments or high-light irradiance.

reviewed in Niyogi and Truong, 2013). qE formation is strictly dependent on a high $\Delta \mathrm{pH}$ and the PsbS protein but also requires zeaxanthin synthesis (Niyogi et al., 1998; Avenson et al., 2008; Holzwarth et al., 2009). Another NPQ component (qZ type quenching), which is distinguished from $\mathrm{qE}$, is formed within 10-30 min (Nilkens et al., 2010). The formation of $\mathrm{qZ}$ is strictly dependent on zeaxanthin but independent of PsbS (Dall'Osto, 2005). The relaxation of $\mathrm{qZ}$ depends on zeaxanthin epoxidation and is linked to the kinetics of the zeaxanthin pool. Photoinhibitory quenching is a zeaxanthinmediated, but not rapidly reversible NPQ component (qI-type quenching or inflexible/sustained thermal dissipation) (Demmig-Adams et al., 2006; reviewed in Pinnola and Bassi, 2018). The relative contribution of each process to the overall NPQ capacity depends on individual photosynthetic organisms and the changing environmental conditions.

Because photosynthetic organisms live in a broad range of habitats, the intensity and spectra of light experienced by different photosynthetic organisms vary extensively. This is particularly true for aquatic organisms, which are subjected to rapidly changing environmental factors, such as abrupt wave movements or phytoplankton migrations. Therefore, it is not surprising that aquatic photosynthetic organisms display distinct photoprotective strategies. For example, the green alga Chlamydomonas reinhardtii (a unicellular model species) and diatom Phaeodactylum tricornutum both need the LHCSR (LHC STRESS-RELATED PROTEIN) family protein for NPQ formation. Synthesis of the Chlamydomonas LHCSR protein is dramatically induced by high light, and it is responsible for the majority of flexible NPQ (Peers et al., 2009; Girolomoni et al., 2019). Notably, the induction of LHCSR expression under high light intensities is found to be controlled by the blue-light photoreceptor phototropin.
This suggests that sensing, dissipation, and utilization of light is a concerted process (Petroutsos et al., 2016). Likewise, the LHCSR family protein LHCX1 in Phaeodactylum determines NPQ's high capacity, which correlates with its strong ability to cope with various light stresses (Bailleul et al., 2010; Gundermann et al., 2019). However, the expression and accumulation of LHCX1 is not further induced by excess light energy like it is with Chlamydomonas LHCSR (Petroutsos et al., 2016). This demonstrates that Phaeodactylum has constitutive and highly efficient photoprotection. These differences in photoprotective capacity between the two aquatic groups reflect their unique ecological adaptations to the sudden, strong changes in underwater light environments.

NPQ mechanisms in terrestrial plants are diverse and elaborate, as reflected by the remarkable diversity of plant species that are distributed in different geographic locations with potentially differential ecological effects. The PsbS (PHOTOSYSTEM II SUBUNIT S) protein in higher plants plays a similar role to the algal type LHCSR. It senses the $\mathrm{pH}$ of the chloroplast thylakoid lumen when there is excess light and induces flexible NPQ (Niyogi et al., 1998; Li et al., 2004; Liguori et al., 2019). Short-lived, fast-growing plants such as annual crops have lower $\mathrm{qE}$ capacity than long-lived, slow-growing species such as tropical evergreens (DemmigAdams et al., 2006). It is possible that slow-growing species utilize a smaller proportion of solar energy for photosynthesis, thus having lower intrinsic photosynthetic capacities. In contrast, in overwintering evergreen plants, $\mathrm{qI}$ is the predominant NPQ component and it operates independent of PsbS and transthylakoid $\mathrm{pH}$, which evolved to cope with combined environmental stresses. The component responsible for $\mathrm{qI}$ is correlated initially with sustained D1 protein phosphorylation 
and xanthophyll cycle arrest and subsequently with newly synthesized zeaxanthin and lutein (Demmig-Adams et al., 2006). This sustained NPQ has critical ecophysiological significance of conferring unique and highly efficient photoprotection in repeated unfavorable seasons over the lifetime of evergreens. It enables these species to downregulate photosynthetic efficiency while continuously harvesting light that does not need to be immediately rechanneled for photosynthesis and growth (reviewed in Demmig-Adams and Adams, 2006). To distinguish the slowly reversible, photoprotective NPQ from qI, this sustained NPQ is termed as $\mathrm{qH}$, which recently has been unveiled to occur in the peripheral antenna of PSII at thylakoid membranes (reviewed in Malnoë, 2018; Malnoë et al., 2018). Genetic screening in Arabidopsis discovered that the molecular player of $\mathrm{qH}$ is the plastid lipocalin, LCNP (Brooks et al., 2013; Malnoë et al., 2018). Intriguingly, LCNP is a soluble protein localized in the thylakoid lumen, whose expression is induced by stresses such as drought or high light (Levesque-Tremblay et al., 2009). These data suggest that the localization of LCNP to thylakoid membranes likely depends on changes in environments (reviewed in Malnoë, 2018). The importance of sustained quenching is to maintain the normal function of thylakoids by allowing photoprotective NPQ in LHCII under stressful conditions (Lacour et al., 2018; Malnoë et al., 2018).

\section{Coevolution of Flexible Non-photochemical Quenching and Antenna in Photosynthetic Lineages}

The wide distribution of NPQ across photosynthetic prokaryotes and eukaryotes highlights its crucial role in PSII photoprotection. Notably, different NPQ systems have evolved in these diverse photosynthetic organisms. Flexible NPQ (qE), the major and also best-studied component of photoprotective excess energy dissipation, constitutes three systems, which are classified based on their associations with the diversification of the lightharvesting equipment in photosynthetic organisms: the OCP (ORANGE CAROTENOID PROTEIN)-dependent system in cyanobacteria, the LHCSR-dependent system in algae and mosses, and the PsbS-dependent system in mosses and vascular plants (Li et al., 2000; Gerotto et al., 2012; Kosuge et al., 2018; Girolomoni et al., 2019; reviewed in Wilson et al., 2006; Rochaix and Bassi, 2019). Therefore, distinct NPQ regulatory mechanisms have evolved to adjust to differential demands of light energy absorption and utilization, allowing ecological adaptations to specific environments. Intriguingly, these diverse types of NPQ are relevant to the diversified antenna systems during evolution of oxygenic photosynthesis. Cyanobacteria deploy thylakoid membrane-bound phycobilisomes as their light-harvesting antenna (reviewed in Kirilovsky and Kerfeld, 2016) and a special carotenoid molecule within OCP to absorb blue-green light and quench excessive excitation energy from phycobilisomes (Wilson et al., 2006; Mezzetti et al., 2019). Cyanobacterial OCP is both the sensor and site of flexible NPQ (Sedoud et al., 2014; Slonimskiy et al., 2019). Algae and plants utilize transmembrane three-helix LHC antennas, which further diversified into algae- and moss-specific LHCSR proteins. Unlike LHC antennas, LHCSR proteins do not absorb light energy but rather act as quenchers by sensing $\mathrm{pH}$ across thylakoid membranes and triggering excess light energy dissipation (Bonente et al., 2011; Pinnola et al., 2013; Tian et al., 2019). In an independent evolutionary innovation from the LHC superfamily, the four-helix protein PsbS in vascular plants functions specifically as a thylakoid membrane $\mathrm{pH}$ sensor to trigger and accelerate the formation of NPQ within the LHC antenna (Li et al., 2000; reviewed in Niyogi and Truong, 2013). In contrast to LHCSR (Bonente et al., 2011; Liguori et al., 2019), PsbS neither binds pigments nor quenches excess excitation energy (Bonente et al., 2008; Ruban et al., 2009; Wilk et al., 2013). Therefore, the sensor (PsbS) and the site (LHC) of NPQ are separated in higher plants, which allow high plasticity and flexibility in efficient NPQ induction and recovery.

It should be mentioned that algae also contains PsbS but only accumulates transiently during high light stress, contrasting with LHCSR that accumulates over a much longer period. PsbS shows the ability to increase NPQ but no clear photoprotection activity (Tibiletti et al., 2016). PsbS is unable to compensate for the function of LHCSR in the lhcsr mutant (Correa-Galvis et al., 2016). LHCSR alone can explain almost all fast induced NPQ in high light acclimated Chlamydomonas cells (Peers et al., 2009). Moss represents a transitional state between algae and vascular plants and has both PSBS and LHCSR. PSBS- and LHCSR-dependent NPQ operate independently and additively (Alboresi et al., 2010; Gerotto et al., 2012). An increased need for flexible NPQ might explain why both LHCSR- and PSBS-dependent NPQ systems are present in early land plants like mosses (Gerotto et al., 2011).

\section{Exploiting Natural Non-photochemical Quenching Variation to Optimize Photoprotection and Photochemical Efficiency}

Natural variation in NPQ capacity is commonly observed in oxygenic photosynthetic organisms, from cyanobacteria to flowering plants, and even between different populations or accessions of the same species grown in the same conditions (Demmig-Adams, 1998; Demmig-Adams et al., 2006; Wang et al., 2017; Hamdani et al., 2019). For instance, different Arabidopsis thaliana ecotypes exhibit diverse maximum levels of NPQ: Col-0 and Ws possess lower NPQ compared to Ll-1, Sf-2 (Jung and Niyogi, 2009). The variations in NPQ are not attributable to differences in PsbS or carotenoids required for NPQ formation but to previously unknown polygenic nuclear traits (Jung and Niyogi, 2009). Identification of these genes and understanding the physiological mechanisms responsible for the high NPQ phenotypes should provide a more complete picture of various NPQ systems and potentially lead to approaches for engineering or breeding plants with enhanced photoprotection capability against adverse environmental conditions while maintaining optimal photosynthetic efficiency. 


\section{OPERATION OF EFFICIENT PHOTOSYSTEM II REPAIR CYCLE ALLOWS HIGH PHOTOSYNTHETIC CAPACITY}

Susceptibility to light-induced photodamage and/or photoinhibition, which can be measured as an increase in NPQ component qI, is an intrinsic and unavoidable feature of all PSII reaction centersfrom cyanobacteria to flowering plants. The main site of photodamage in PSII is the reaction center D1 subunit, which constantly undergoes rapid turnover (degradation and synthesis) (Aro et al., 1993; Reviewed in Järvi et al., 2015). Although cyanobacteria, algae, and plants have repair mechanisms that differ in detail, they share a central feature: the replacement of the photodamaged D1 subunit with a newly synthesized copy (Armbruster et al., 2010; Kato et al., 2012; reviewed in Nixon et al., 2005; Komenda et al., 2012; $\mathrm{Lu}, 2016)$. The PSII repair cycle involves disassembly, targeted reaction-center protein proteolysis, replacement of damaged core proteins, and reassembly of new functional PSII supercomplexes (Haußühl et al., 2001; Kato et al., 2018; reviewed in Nickelsen and Rengstl, 2013). In addition, individual steps in the PSII repair cycle are vulnerable to environmental changes (reviewed in Nath et al., 2013), further necessitating an efficient and timely operation of the repair machinery (Figure 1).

Cyanobacteria and chloroplasts employ distinct PSII repair mechanisms, which may be relevant to evolutionarily distinct thylakoid structures. The photosynthetic membrane systems in oxygenic photosynthetic organisms have evolved into discrete morphological architectures, despite their common ancestryeukaryotic chloroplasts evolved from cyanobacteria via an ancient endosymbiotic event (reviewed in Ku et al., 2015; Bock, 2017). In plant chloroplasts, photosynthetic membranes are differentiated into a network of extensively stacked grana thylakoids and unstacked stromal lamellae. Grana thylakoids are enriched in functional PSII supercomplexes, while the interconnecting stromal lamellae are enriched in PSI and ATP synthase complexes, with cytochrome $b_{6} f$ complex evenly distributed between the two (Dekker and Boekema, 2005; Daum et al., 2010). In contrast, cyanobacterial thylakoid membranes are not differentiated in grana and stromal lamellae; therefore, their photosynthetic apparatus are not laterally separated (Liberton et al., 2013; Rast et al., 2019).

In higher plants, the individual repair steps take place in discrete subcompartments and occur in a well-defined order (reviewed in Kosuge et al., 2018). Kinases, phosphatases, proteases, ribosomes, and repair/assembly factors are spatially segregated to ensure an operation with minimal interference (Puthiyaveetil et al., 2014; Koochak et al., 2019). Phosphorylation remodels the thylakoid structure to facilitate monomerization of photodamaged PSII supercomplexes in the grana core. These damaged monomeric PSII complexes are then trafficked to granal margins, where dephosphorylation and disassembly likely occur. This allows damaged D1 to be degraded successively by FtSH and Deg proteases (Haußühl et al., 2001; Kato et al., 2012; Krynická et al., 2015; reviewed in Silva et al., 2003; Sun et al., 2007; Tikkanen et al., 2008; Li et al., 2018). The site of de novo D1 protein synthesis is located in unstacked stroma lamellae, whereas reformation of active PSII supercomplexes takes place in the highly stacked grana core (Okada et al., 1996; Danielsson et al., 2006).

The green alga Chlamydomonas has a thylakoid membrane organization similar to that in higher plants, though with less stacking of thylakoid membranes in its single cup-shaped chloroplast (Wei et al., 2014). Consistent with the less stacking of thylakoids, experimental evidence indicates that individual PSII repair steps in Chlamydomonas are not restricted to thylakoid subdomains but rather are dispersed all over in the thylakoids (Uniacke and Zerges, 2007).

PSII repair in cyanobacteria seems to be restricted to specific sites in the thylakoid membranes named repair zones (Silva et al., 2003; Klinkert et al., 2004). Some other studies demonstrated that these repair zones could also be located in the plasma membrane where repair zones converge with PSII biogenesis centers at PDM (PRATA-DEFINED MEMBRANES) subcompartments to allow damaged D1 to be promptly replaced (Schottkowski et al., 2009; Stengel et al., 2012). Another special feature in cyanobacteria is that the conserved phosphorylatable threonine residues in PSII reaction center proteins are not phosphorylated during PSII repair (Calzadilla et al., 2019; reviewed in Komenda et al., 2012). This suggests that phosphorylation- and dephosphorylation-facilitated PSII repair may be a specific step evolved in photosynthetic eukaryotes.

\section{APPROPRIATE PHOTOSYSTEM II MAINTENANCE ENSURES OPTIMAL PHOTOSYNTHETIC PERFORMANCE UNDER NATURAL FLUCTUATING LIGHT ENVIRONMENTS}

Photosynthetic organisms experience abrupt and strong changes in light irradiance from seconds to seasons in their aquatic or terrestrial habitats. A multitude of protective and regulatory mechanisms evolved to facilitate their adaptation to such environmental fluctuations. NPQ appears to be a ubiquitous and major light acclimation mechanism that contributes to fitness under varying environments. LHCSR deficiency caused an increased death rate in Chlamydomonas following a shift from low to high light, suggesting that LHCSR-induced NPQ is required for optimal survival under variable light conditions (Peers et al., 2009; Kosuge et al., 2018; Girolomoni et al., 2019; Tian et al., 2019). In Phaeodactylum, a decreased LHCX1 level led to reduced fitness under stressful light, and even non-stressful light conditions, suggesting that LHCX-dependent NPQ endows diatoms with maximal survival capacity under a wide range of light environments (Bailleul et al., 2010; Gundermann et al., 2019; Park et al., 2019). In Arabidopsis plants, NPQ plays a crucial role in rapidly adjusting PSII to artificial fluctuating light (Armbruster et al., 2014, 2016; Duan et al., 2016; Herdean et al., 2016). In field conditions with natural fluctuating light, the NPQ-defective mutants $n p q 1$ and npq4 exhibited lower PSII activity and produced fewer seeds than the wild type, although they had no visible vegetative growth defects (reviewed in Külheim et al., 2002; Frenkel et al., 2007; 
Wobbe et al., 2016). Compared to what we know of NPQ in algae and plants, little is known about the importance of OCP-dependent NPQ in cyanobacteria under fluctuating light.

So far, there is no published experimental evidence addressing whether PSII deficiency affects cyanobacteria or Chlamydomonas growth under variable light conditions, but several studies in Arabidopsis identified protein factors required to safeguard PSII under rapidly changing light conditions. TLP18.3 (THYLAKOID LUMEN PROTEIN 18.3) protein is reported to have a crucial role in adjusting Arabidopsis photosynthesis to fluctuating light (Sirpio et al., 2007; Jarvi et al., 2016). The tlp18.3 mutants did not show visible phenotype under standard growth conditions. However, they exhibited retarded growth under fluctuating light and were highly susceptible to high-light stress. More importantly, the phenotypic defects of the tlp18.3 mutants were found to be associated with inefficient operation of the PSII repair cycle (Sirpio et al., 2007). Two recent studies uncovered that the loss of Arabidopsis PSB27 (PHOTOSYSTEM II SUBUNIT 27) and MET1 (MESOPHYLL-ENRICHED THYLAKOID PROTEIN 1) caused stunted phenotypes when exposed to fluctuating light intensities (Bhuiyan et al., 2015; Hou et al., 2015). These lossof-function mutations did not affect growth and development under normal light conditions. The reduced vegetative growth in the psb27 mutant under fluctuating light was attributed to decreased PSII efficiency; this, however, was independent to the PSII supercomplex formation (Hou et al., 2015). The growth retardation in met 1 was due to a defect in the regeneration of active PSII supercomplexes that correlated with the reduced PSII activity (Bhuiyan et al., 2015). Other PSII repair-impaired mutants, including the newly characterized $m p h 2$ and curt1, displayed growth retardation under fluctuating light (Liu and Last, 2017; Pribil et al., 2018). The association of decreased growth with impairments in PSII repair suggests that proper maintenance of PSII photochemical efficiency represents an important strategy to ensure plant fitness under adverse light conditions. Exploring the mechanisms of PSII repair in algae and cyanobacteria under fluctuating light may offer further insight into the evolution of photosynthesis. Moreover, exploiting PSII repair mechanisms could be promising targets for bioengineering photosynthesis to increase photosynthetic capacity and productivity under controlled photoinhibitory light and natural fluctuating light environments.

\section{OPTIMIZING NON-PHOTOCHEMICAL QUENCHING TO ENHANCE PHOTOSYNTHETIC CAPACITY AND GROWTH IN FIELD CONDITIONS}

Deeper understanding of NPQ mechanisms should inform strategies to optimize the balance between photoprotection and photosynthetic productivity. Optimization of photoprotection to improve photosynthetic performance is an emerging strategy in agriculture. It is generally accepted that the solar energy conversion efficiency for crop plants is much lower than the theoretical maximum yield ( 12\%) (reviewed in Walker, 2009; Blankenship et al., 2011). One major cause for the low efficiency is that upper leaves of a canopy absorb more sunlight than can be used for photochemistry, while photosynthesis of lower leaves is limited by shading (reviewed in Long et al., 2015). Altering the pigment content and leaf arrangement in the canopy may improve crop yield. A smart canopy with even light absorption would have light green vertical leaves at the top of the canopy and dark green horizontal leaves at the bottom (reviewed in Ort et al., 2015). Therefore, an optimized canopy may achieve higher crop yield.

Another major reason for the lower than the expected maximal photosynthetic efficiency in crops (and other plants) is that NPQ relaxation lags behind fluctuations in sunlight during sudden transitions from high to low light. This happens when passing clouds or movement of neighboring leaves/plant species shade sunlit leaves. The slow NPQ response could cost up to $30 \%$ of carbon gain (Zhu et al., 2004, reviewed in Zhu et al., 2008), suggesting that accelerating NPQ relaxation would be a strategy for increasing photosynthetic productivity. For example, speeding up the response to natural shading events by enhancing the recovery from photoprotective NPQ in Nicotiana tabaccum markedly increased photosynthetic capacity and bulked up leaves, stems, and roots, which contributed to a $15 \%$ gain in plant biomass production in field conditions (Kromdijk et al., 2016). Much more rapid NPQ induction in bright light and much faster NPQ relaxation following a drop in light intensity enable plants to track fluctuations in sunlight more closely, contributing to more efficient light energy utilization and carbon fixation. This proofof-concept field trial opens the door to enhancing photosynthetic performance and productivity in agricultural and natural ecosystems.

\section{CONCLUDING REMARKS AND FUTURE PERSPECTIVES}

In oxygenic photosynthesis, it is important to (1) safely handle excess absorbed light energy that would otherwise cause massive ROS production and damage the photosynthetic machinery and (2) efficiently convert solar energy into chemical bond energy. Tight regulation of these two aspects may contribute to an increase in productivity in agriculture and natural ecosystems. Understanding the elaborate NPQ mechanisms and the robust PSII repair systems may help identify targets to optimize photosynthetic efficiency. This would facilitate translational work toward exploring yield potential to sustainably meet the global rising demands for food, fuel, and fiber in the future climate change. Prior to accomplishing these grand goals, multiple outstanding questions await to be addressed:

1. Do antenna-based photoprotection and reaction center-based repair operate in concert or in parallel to regulate PSII efficiency and photosynthetic capacity under photoinhibitory light and other environmental stresses? How does evolution of NPQ in the oxygenic organisms contribute to that of repair and vice versa?

2. How do ROS regulate PSII activity under fluctuating light environments or field conditions?

3. Are the molecular mechanisms of PSII repair under changing light different or similar to those under high-light irradiance? Can photosynthetic species discern PSII damage caused by 
these two types of light conditions and initiate distinct repair strategies?

\section{AUTHOR CONTRIBUTIONS}

JL and RL conceived the project. JL, YL, WH, and RL wrote and edited the manuscript.

\section{REFERENCES}

Alboresi, A., Dall'Osto, L., Aprile, A., Carillo, P., Roncaglia, E., Cattivelli, L., et al. (2011). Reactive oxygen species and transcript analysis upon excess light treatment in wild-type Arabidopsis thaliana vs a photosensitive mutant lacking zeaxanthin and lutein. BMC Plant Biol. 11:62. doi: 10.1186/1471-2229-11-62

Alboresi, A., Gerotto, C., Giacometti, G. M., Bassi, R., and Morosinotto, T. (2010). Physcomitrella patens mutants affected on heat dissipation clarify the evolution of photoprotection mechanisms upon land colonization. Proc. Natl. Acad. Sci. USA 107, 11128-11233. doi: 10.1073/pnas.1002873107

Armbruster, U., Carrillo, L. R., Venema, K., Pavlovic, L., Schmidtmann, E., Kornfeld, A., et al. (2014). Ion antiport accelerates photosynthetic acclimation in fluctuating light environments. Nat. Commun. 5:5439. doi: 10.1038/ ncomms6439

Armbruster, U., Leonelli, L., Correa Galvis, V., Strand, D., Quinn, E. H., Jonikas, M. C., et al. (2016). Regulation and levels of the thylakoid $\mathrm{K}+\mathrm{H}+$ antiporter KEA3 shape the dynamic response of photosynthesis in fluctuating light. Plant Cell Physiol. 57, 1557-1567. doi: 10.1093/pcp/pcw085

Armbruster, U., Zühlke, J., Rengstl, B., Kreller, R., Makarenko, E., Rühle, T., et al. (2010). The Arabidopsis thylakoid protein PAM68 is required for efficient D1 biogenesis and photosystem II assembly. Plant Cell 22, 3439-3460. doi: $10.1105 /$ tpc. 110.077453

Aro, E. M., Virgin, I., and Andersson, B. (1993). Photoinhibition of photosystem II. Inactivation, protein damage and turnover. Biochim. Biophys. Acta Bioenerg. 1143, 113-134. doi: 10.1016/0005-2728(93)90134-2

Avenson, T. J., Tae, K. A., Zigmantas, D., Niyogi, K. K., Li, Z., Ballottari, M., et al. (2008). Zeaxanthin radical cation formation in minor light-harvesting complexes of higher plant antenna. J. Biol. Chem. 283, 3550-3558. doi: 10.1074/jbc.M705645200

Bailleul, B., Rogato, A., de Martino, A., Coesel, S., Cardol, P., Bowler, C., et al. (2010). An atypical member of the light-harvesting complex stress-related protein family modulates diatom responses to light. Proc. Natl. Acad. Sci. USA 107, 18214-18219. doi: 10.1073/pnas.1007703107

Bellafiore, S., Barneche, F., Peltier, G., and Rochaix, J. D. (2005). State transitions and light adaptation require chloroplast thylakoid protein kinase STN7. Nature 433, 892-895. doi: 10.1038/nature03286

Bhuiyan, N. H., Friso, G., Poliakov, A., Ponnala, L., and van Wijk, K. J. (2015). MET1 Is a thylakoid-associated TPR protein involved in photosystem II supercomplex formation and repair in Arabidopsis. Plant Cell 27, 262-285. doi: $10.1105 /$ tpc.114.132787

Blankenship, R. E., Tiede, D. M., Barber, J., Brudvig, G. W., Fleming, G., Ghirardi, M., et al. (2011). Comparing photosynthetic and photovoltaic efficiencies and recognizing the potential for improvement. Science 332, 805-809. doi: $10.1126 /$ science. 1200165

Bock, R. (2017). Witnessing genome evolution: experimental reconstruction of endosymbiotic and horizontal gene transfer. Annu. Rev. Genet. 51, 1-22. doi: 10.1146/annurev-genet-120215-035329

Bonente, G., Ballottari, M., Truong, T. B., Morosinotto, T., Ahn, T. K., Fleming, G. R., et al. (2011). Analysis of LhcSR3, a protein essential for feedback de-excitation in the green alga Chlamydomonas reinhardtii. PLoS Biol. 9:e1000577. doi: 10.1371/journal.pbio.1000577

Bonente, G., Howes, B. D., Caffarri, S., Smulevich, G., and Bassi, R. (2008). Interactions between the photosystem II subunit PsbS and xanthophylls studied in vivo and in vitro. J. Biol. Chem. 283, 8434-8445. doi: 10.1074/ jbc.M708291200

\section{FUNDING}

The National Key Basic Research Program of China (2015CB150200), National Natural Science Foundation of China (31800196) and Fundamental Research Funds for Central Non-profit Scientific Institution (1610172019006) supported this work. YL and RL acknowledge funding from the US National Science Foundation (MCB-1244008).

Brooks, M. D., Sylak-Glassman, E. J., Fleming, G. R., and Niyogi, K. K. (2013) A thioredoxin-like/-propeller protein maintains the efficiency of light harvesting in Arabidopsis. Proc. Natl. Acad. Sci. USA 110, 2733-2740. doi: 10.1073/ pnas. 1305443110

Calzadilla, P. I., Zhan, J., Sétif, P., Lemaire, C., Solymosi, D., Battchikova, N., et al. (2019). The cytochrome $b_{6} f$ complex is not involved in cyanobacterial state transitions. Plant Cell 31, 911-931. doi: 10.1105/tpc.18.00916

Chen, Y.-E., Su, Y.-Q., Mao, H.-T., Wu, N., Zhu, F., Yuan, M., et al. (2018). Terrestrial plants evolve highly assembled photosystem complexes in adaptation to light shifts. Front. Plant Sci. 9:1811. doi: 10.3389/fpls.2018.01811

Chen, Y.-E., Yuan, S., Lezhneva, L., Meurer, J., Schwenkert, S., Mamedov, F., et al. (2019). The low molecular mass photosystem II protein PsbTn is important for light acclimation. Plant Physiol. 79, 1739-1753. doi: 10.1104/ pp.18.01251

Correa-Galvis, V., Redekop, P., Guan, K., Griess, A., Truong, T. B., Wakao, S. et al. (2016). Photosystem II subunit PsbS is involved in the induction of LHCSR protein-dependent energy dissipation in Chlamydomonas reinhardtii. J. Biol. Chem. 291, 17478-17487. doi: 10.1074/jbc.M116.737312

Dall'Osto, L. (2005). A mechanism of nonphotochemical energy dissipation, independent from PsbS, revealed by a conformational change in the antenna protein CP26. Plant Cell 17, 1217-1232. doi: 10.1105/tpc.104.030601

Danielsson, R., Suorsa, M., Paakkarinen, V., Albertsson, P. Å., Styring, S., Aro, E. M., et al. (2006). Dimeric and monomeric organization of photosystem II: distribution of five distinct complexes in the different domains of the thylakoid membrane. J. Biol. Chem. 281, 14241-14249. doi: 10.1074/jbc.M600634200

Daum, B., Nicastro, D., Austin, J., McIntosh, J. R., and Kühlbrandt, W. (2010). Arrangement of photosystem II and ATP synthase in chloroplast membranes of spinach and pea. Plant Cell 22, 1299-1312. doi: 10.1105/tpc.109.071431

Dekker, J. P., and Boekema, E. J. (2005). Supramolecular organization of thylakoid membrane proteins in green plants. Biochim. Biophys. Acta Bioenerg. 1706, 12-39. doi: 10.1016/j.bbabio.2004.09.009

Demmig-Adams, B. (1998). Survey of thermal energy dissipation and pigment composition in sun and shade leaves. Plant Cell Physiol. 39, 474-482. doi: 10.1093/oxfordjournals.pcp.a029394

Demmig-Adams, B., and Adams, W. W. (2006). Photoprotection in an ecological context: the remarkable complexity of thermal energy dissipation. New Phytol. 172, 11-21. doi: 10.1111/j.1469-8137.2006.01835.x

Demmig-Adams, B., Ebbert, V., Mellman, D. L., Mueh, K. E., Schaffer, L., Funk, C., et al. (2006). Modulation of PsbS and flexible vs sustained energy dissipation by light environment in different species. Physiol. Plant. 127, 670-680. doi: 10.1111/j.1399-3054.2006.00698.x

Dogra, V., Rochaix, J. D., and Kim, C. (2018). Singlet oxygen-triggered chloroplastto-nucleus retrograde signalling pathways: an emerging perspective. Plant Cell Environ. 41, 1727-1738. doi: 10.1111/pce.13332

Duan, Z., Kong, F., Zhang, L., Li, W., Zhang, J., and Peng, L. (2016). A bestrophin-like protein modulates the proton motive force across the thylakoid membrane in Arabidopsis. J. Integr. Plant Biol. 58, 848-858. doi: 10.1111/ jipb. 12475

Erickson, E., Wakao, S., and Niyogi, K. K. (2015). Light stress and photoprotection in Chlamydomonas reinhardtii. Plant J. 82, 449-465. doi: $10.1111 /$ tpj.12825

Frenkel, M., Bellafiore, S., Rochaix, J. D., and Jansson, S. (2007). Hierarchy amongst photosynthetic acclimation responses for plant fitness. Physiol. Plant. 129, 455-459. doi: 10.1111/j.1399-3054.2006.00831.x

Frigerio, S., Campoli, C., Zorzan, S., Fantoni, L. I., Crosatti, C., Drepper, F., et al. (2007). Photosynthetic antenna size in higher plants is controlled by 
the plastoquinone redox state at the post-transcriptional rather than transcriptional level. J. Biol. Chem. 282, 29457-29469. doi: 10.1074/jbc. M705132200

Gerotto, C., Alboresi, A., Giacometti, G. M., Bassi, R., and Morosinotto, T. (2011). Role of PSBS and LHCSR in Physcomitrella patens acclimation to high light and low temperature. Plant Cell Environ. 34, 922-932. doi: 10.1111/j. 1365-3040.2011.02294.x

Gerotto, C., Alboresi, A., Giacometti, G. M., Bassi, R., and Morosinotto, T. (2012). Coexistence of plant and algal energy dissipation mechanisms in the moss Physcomitrella patens. New Phytol. 196, 763-773. doi: 10.1111/j.1469-8137.2012.04345.x

Ghotbi-Ravandi, A. A., Shahbazi, M., Shariati, M., and Mulo, P. (2014). Effects of mild and severe drought stress on photosynthetic efficiency in tolerant and susceptible barley (Hordeum vulgare L.) genotypes. J. Agron. Crop Sci. 200, 403-415. doi: 10.1111/jac.12062

Girolomoni, L., Cazzaniga, S., Pinnola, A., Perozeni, F., Ballottari, M., and Bassi, R. (2019). LHCSR3 is a nonphotochemical quencher of both photosystems in Chlamydomonas reinhardtii. Proc. Natl. Acad. Sci. USA 116, 4212-4217. doi: 10.1073/pnas.1809812116

Gundermann, K., Wagner, V., Mittag, M., and Büchel, C. (2019). Fucoxanthinchlorophyll protein complexes of the centric diatom Cyclotella meneghiniana differ in Lhcx1 and Lhcx6 content. Plant Physiol. 179, 1779-1795. doi: 10.1104/pp.18.01363

Hamdani, S., Khan, N., Perveen, S., Qu, M., Jiang, J., Govindjee et al. (2019). Changes in the photosynthesis properties and photoprotection capacity in rice (Oryza sativa) grown under red, blue, or white light. Photosynth. Res. 139, 107-121. doi: 10.1007/s11120-018-0589-6

Haußühl, K., Andersson, B., and Adamska, I. (2001). A chloroplast DegP2 protease performs the primary cleavage of the photodamaged D1 protein in plant photosystem II. EMBO J. 20, 713-722. doi: 10.1093/emboj/20.4.713

Herdean, A., Teardo, E., Nilsson, A. K., Pfeil, B. E., Johansson, O. N., Unnep, R., et al. (2016). A voltage-dependent chloride channel fine-tunes photosynthesis in plants. Nat. Commun. 7:11654. doi: 10.1038/ncomms11654

Hey, D., and Grimm, B. (2018). One-helix protein 2 (OHP2) is required for the stability of OHP1 and assembly factor HCF244 and is functionally linked to PSII biogenesis. Plant Physiol. 177, 1453-1472. doi: 10.1104/ pp. 18.00540

Holzwarth, A. R., Miloslavina, Y., Nilkens, M., and Jahns, P. (2009). Identification of two quenching sites active in the regulation of photosynthetic lightharvesting studied by time-resolved fluorescence. Chem. Phys. Lett. 483, 262-267. doi: 10.1016/j.cplett.2009.10.085

Hou, X., Fu, A., Garcia, V. J., Buchanan, B. B., and Luan, S. (2015). PSB27: a thylakoid protein enabling Arabidopsis to adapt to changing light intensity. Proc. Natl. Acad. Sci. USA 112, 1613-1618. doi: 10.1073/pnas.1424040112

Jarillo, J. A., Gabrys, H., Capel, J., Alonso, J. M., Ecker, J. R., and Cashmore, A. R. (2001). Phototropin-related NPL1 controls chloroplast relocation induced by blue light. Nature 410, 952-954. doi: 10.1038/35073622

Jarvi, S., Isojarvi, J., Kangasjarvi, S., Salojarvi, J., Mamedov, F., Suorsa, M., et al. (2016). Photosystem II repair and plant immunity: lessons learned from Arabidopsis mutant lacking the THYLAKOID LUMEN PROTEIN 18.3. Front. Plant Sci. 7:405. doi: 10.3389/fpls.2016.00405

Järvi, S., Suorsa, M., and Aro, E. M. (2015). Photosystem II repair in plant chloroplasts-regulation, assisting proteins and shared components with photosystem II biogenesis. Biochim. Biophys. Acta Bioenerg. 1847, 900-909. doi: 10.1016/j.bbabio.2015.01.006

Jin, H. L., Liu, B., Luo, L. J., Feng, D. R., Wang, P., Liu, J., et al. (2014). Hypersensitive to high LIGHT1 interacts with low quantum yield of photosystem II1 and functions in protection of photosystem II from photodamage in Arabidopsis. Plant Cell 26, 1213-1229. doi: 10.1105/tpc.113.122424

Jung, H., and Niyogi, K. K. (2009). Quantitative genetic analysis of thermal dissipation in Arabidopsis. Plant Physiol. 150, 977-986. doi: 10.1104/ pp.109.137828

Kale, R., Hebert, A. E., Frankel, L. K., Sallans, L., Bricker, T. M., and Pospiśsil, P. (2017). Amino acid oxidation of the D1 and D2 proteins by oxygen radicals during photoinhibition of photosystem II. Proc. Natl. Acad. Sci. USA 114, 2988-2993. doi: 10.1073/pnas.1618922114

Kapri-Pardes, E., Naveh, L., and Adam, Z. (2007). The thylakoid lumen protease Deg1 is involved in the repair of photosystem II from photoinhibition in Arabidopsis. Plant Cell 19, 1039-1047. doi: 10.1105/tpc.106.046573
Kato, Y., Hyodo, K., and Sakamoto, W. (2018). The photosystem II repair cycle requires FtsH turnover through the EngA GTPase. Plant Physiol. 178, 596-611. doi: 10.1104/pp.18.00652

Kato, Y., Sun, X., Zhang, L., and Sakamoto, W. (2012). Cooperative D1 degradation in the photosystem II repair mediated by chloroplastic proteases in Arabidopsis. Plant Physiol. 159, 1428-1439. doi: 10.1104/pp.112.199042

Kern, J., and Renger, G. (2007). Photosystem II: structure and mechanism of the water: plastoquinone oxidoreductase. Photosynth. Res. 94, 183-202. doi: 10.1007/s11120-007-9201-1

Kirilovsky, D., and Kerfeld, C. A. (2016). Cyanobacterial photoprotection by the orange carotenoid protein. Nat. Plants 2, 1-7. doi: 10.1038/nplants.2016.180

Klinkert, B., Ossenbühl, F., Sikorski, M., Berry, S., Eichacker, L., and Nickelsen, J. (2004). PratA, a periplasmic tetratricopeptide repeat protein involved in biogenesis of photosystem II in Synechocystis sp. PCC 6803. J. Biol. Chem. 279, 44639-44644. doi: 10.1074/jbc.M405393200

Komenda, J., Sobotka, R., and Nixon, P. J. (2012). Assembling and maintaining the photosystem II complex in chloroplasts and cyanobacteria. Curr. Opin. Plant Biol. 15, 245-251. doi: 10.1016/j.pbi.2012.01.017

Koochak, H., Puthiyaveetil, S., Mullendore, D. L., Li, M., and Kirchhoff, H. (2019). The structural and functional domains of plant thylakoid membranes. Plant J. 97, 412-429. doi: 10.1111/tpj.14127

Kosuge, K., Tokutsu, R., Kim, E., Akimoto, S., Yokono, M., Ueno, Y., et al. (2018). LHCSR1-dependent fluorescence quenching is mediated by excitation energy transfer from LHCII to photosystem I in Chlamydomonas reinhardtii. Proc. Natl. Acad. Sci. USA 115, 3722-3727. doi: 10.1073/pnas.1720574115

Kromdijk, J., Głowacka, K., Leonelli, L., Gabilly, S., Iwai, M., Niyogi, K., et al. (2016). Improving photosynthesis and crop productivity by accelerating recovery from photoprotection. Science 354, 857-861. doi: 10.1126/science. aai8878

Krynická, V., Shao, S., Nixon, P. J., and Komenda, J. (2015). Accessibility controls selective degradation of photosystem II subunits by FtsH protease. Nat. Plants 1:15168. doi: 10.1038/nplants.2015.168

Ku, C., Nelson-Sathi, S., Roettger, M., Sousa, F. L., Lockhart, P. J., Bryant, D., et al. (2015). Endosymbiotic origin and differential loss of eukaryotic genes. Nature 524, 427-432. doi: 10.1038/nature14963

Külheim, C., Agren, J., and Jansson, S. (2002). Rapid regulation of light harvesting and plant fitness in the field. Science 297, 91-93. doi: 10.1126/science.1072359

Lacour, T., Larivière, J., Ferland, J., Bruyant, F., Lavaud, J., and Babin, M. (2018). The role of sustained photoprotective non-photochemical quenching in low temperature and high light acclimation in the bloom-forming arctic diatom Thalassiosira gravida. Front. Mar. Sci. 5:354. doi: 10.3389/ fmars.2018.00354

Leister, D. (2019). Genetic engineering, synthetic biology and the light reactions of photosynthesis. Plant Physiol. 179, 778-793. doi: 10.1104/pp.18.00360

Levesque-Tremblay, G., Havaux, M., and Ouellet, F. (2009). The chloroplastic lipocalin AtCHL prevents lipid peroxidation and protects Arabidopsis against oxidative stress. Plant J. 60, 691-702. doi: 10.1111/j.1365-313X.2009.03991.x

Li, L., Aro, E. M., and Millar, A. H. (2018). Mechanisms of photodamage and protein turnover in photoinhibition. Trends Plant Sci. 23, 667-676. doi: 10.1016/j.tplants.2018.05.004

Li, X. P., Bjorkman, O., Shih, C., Grossman, A. R., Rosenquist, M., Jansson, S., et al. (2000). A pigment-binding protein essential for regulation of photosynthetic light harvesting. Nature 403, 391-395. doi: 10.1038/35000131

Li, X. P., Gilmore, A. M., Caffarri, S., Bassi, R., Golan, T., Kramer, D., et al. (2004). Regulation of photosynthetic light harvesting involves intrathylakoid lumen $\mathrm{pH}$ sensing by the PsbS protein. J. Biol. Chem. 279, 22866-22874. doi: 10.1074/jbc.M402461200

Li, Y., Liu, B., Zhang, J., Kong, F., Zhang, L., Meng, H., et al. (2019). OHP1, OHP2, and HCF244 form a transient functional complex with the photosystem II reaction center. Plant Physiol. 179, 195-208. doi: 10.1104/ pp.18.01231

Liberton, M., Page, L. E., O’Dell, W. B., O’Neill, H., Mamontov, E., Urban, V. S., et al. (2013). Organization and flexibility of cyanobacterial thylakoid membranes examined by neutron scattering. J. Biol. Chem. 288, 3632-3640. doi: 10.1074/ jbc.M112.416933

Liguori, N., Campos, S. R. R., Baptista, A. M., and Croce, R. (2019). Molecular anatomy of plant photoprotective switches: the sensitivity of PsbS to the environment, residue by residue. J. Phys. Chem. Lett. 10, 1737-1742. doi: 10.1021/acs.jpclett.9b00437 
Liu, J., and Last, R. L. (2015a). A land plant-specific thylakoid membrane protein contributes to photosystem II maintenance in Arabidopsis thaliana. Plant J. 82, 731-743. doi: 10.1111/tpj. 12845

Liu, J., and Last, R. L. (2015b). MPH1 is a thylakoid membrane protein involved in protecting photosystem II from photodamage in land plants. Plant Signal. Behav. 10:e1076602. doi: 10.1080/15592324.2015.1076602

Liu, J., and Last, R. L. (2017). A chloroplast thylakoid lumen protein is required for proper photosynthetic acclimation of plants under fluctuating light environments. Proc. Natl. Acad. Sci. USA 114, E8110-E8117. doi: 10.1073/ pnas. 1712206114

Long, S. P., Marshall-Colon, A., and Zhu, X. G. (2015). Meeting the global food demand of the future by engineering crop photosynthesis and yield potential. Cell 161, 56-66. doi: 10.1016/j.cell.2015.03.019

$\mathrm{Lu}, \mathrm{Y}$. (2011). The occurrence of a thylakoid-localized small zinc finger protein in land plants. Plant Signal. Behav. 6, 1881-1885. doi: 10.4161/psb.6.12.18022

$\mathrm{Lu}$, Y. (2016). Identification and roles of photosystem II assembly, stability, and repair factors in Arabidopsis. Front. Plant Sci. 7:168. doi: 10.3389/ fpls.2016.00168

Lu, Y., Hall, D. A., and Last, R. L. (2011). A small Zinc finger thylakoid protein plays a role in maintenance of photosystem II in Arabidopsis thaliana. Plant Cell 23, 1861-1875. doi: 10.1105/tpc.111.085456

Malnoë, A. (2018). Photoinhibition or photoprotection of photosynthesis? Update on the (newly termed) sustained quenching component qH. Environ. Exp. Bot. 154, 123-133. doi: 10.1016/j.envexpbot.2018.05.005

Malnoë, A., Schultink, A., Shahrasbi, S., Rumeau, D., Havaux, M., and Niyogi, K. K. (2018). The plastid lipocalin LCNP is required for sustained photoprotective energy dissipation in Arabidopsis. Plant Cell 30, 196-208. doi: 10.1105/tpc.17.00536

Mezzetti, A., Alexandre, M., Thurotte, A., Wilson, A., Gwizdala, M., and Kirilovsky, D. (2019). Two-step structural changes in orange carotenoid protein photoactivation revealed by time-resolved Fourier transform infrared spectroscopy. J. Phys. Chem. B 123, 3259-3266. doi: 10.1021/acs.jpcb.9b01242

Mishra, N. P., and Ghanotakis, D. F. (1994). Exposure of a photosystem II complex to chemically generated singlet oxygen results in D1 fragments similar to the ones observed during aerobic photoinhibition. Biochim. Biophys. Acta Bioenerg. 1187, 296-300. doi: 10.1016/0005-2728(94)90003-5

Miyao, M., Ikeuchi, M., Yamamoto, N., and Ono, T. A. (1995). Specific degradation of the D1 protein of photosystem II by treatment with hydrogen peroxide in darkness: implications for the mechanism of degradation of the D1 protein under illumination. Biochemistry 34, 10019-10026. doi: 10.1021/bi00031a025

Murata, N., Takahashi, S., Nishiyama, Y., and Allakhverdiev, S. I. (2007). Photoinhibition of photosystem II under environmental stress. Biochim. Biophys. Acta Bioenerg. 1767, 414-421. doi: 10.1016/j.bbabio.2006.11.019

Myouga, F., Takahashi, K., Tanaka, R., Nagata, N., Kiss, A. Z., Funk, C., et al. (2018). Stable accumulation of photosystem II requires ONE-HELIX PROTEIN1 (OHP1) of the light harvesting-like family. Plant Physiol. 176, 2277-2291. doi: $10.1104 / p p .17 .01782$

Nath, K., Jajoo, A., Poudyal, R. S., Timilsina, R., Park, Y. S., Aro, E. M., et al. (2013). Towards a critical understanding of the photosystem II repair mechanism and its regulation during stress conditions. FEBS Lett. 587, 3372-3381. doi: 10.1016/j.febslet.2013.09.015

Nickelsen, J., and Rengstl, B. (2013). Photosystem II assembly: from cyanobacteria to plants. Annu. Rev. Plant Biol. 64, 609-635. doi: 10.1146/annurevarplant-050312-120124

Nilkens, M., Kress, E., Lambrev, P., Miloslavina, Y., Müller, M., Holzwarth, A. R., et al. (2010). Identification of a slowly inducible zeaxanthin-dependent component of non-photochemical quenching of chlorophyll fluorescence generated under steady-state conditions in Arabidopsis. Biochim. Biophys. Acta Bioenerg. 1794, 466-475. doi: 10.1016/j.bbabio.2010.01.001

Nishiyama, Y., Yamamoto, H., Allakhverdiev, S. I., Inaba, M., Yokota, A., and Murata, N. (2001). Oxidative stress inhibits the repair of photodamage to the photosynthetic machinery. EMBO J. 20, 5587-5594. doi: 10.1093/ emboj/20.20.5587

Nixon, P. J., Barker, M., Boehm, M., De Vries, R., and Komenda, J. (2005). FtsH-mediated repair of the photosystem II complex in response to light stress. J. Exp. Bot. 56, 357-363. doi: 10.1093/jxb/eri021

Niyogi, K. K., Grossman, A. R., and Bjorkman, O. (1998). Arabidopsis mutants define a central role for the xanthophyll cycle in the regulation of photosynthetic energy conversion. Plant Cell 10, 1121-1134. doi: 10.1105/tpc.10.7.1121
Niyogi, K. K., and Truong, T. B. (2013). Evolution of flexible non-photochemical quenching mechanisms that regulate light harvesting in oxygenic photosynthesis. Curr. Opin. Plant Biol. 16, 307-314. doi: 10.1016/j.pbi.2013.03.011

Okada, K., Ikeuchi, M., Yamamoto, N., Ono, T. A., and Miyao, M. (1996). Selective and specific cleavage of the D1 and D2 proteins of photosystem II by exposure to singlet oxygen: factors responsible for the susceptibility to cleavage of the proteins. Biochim. Biophys. Acta Bioenerg. 1274, 73-79. doi: 10.1016/0005-2728(96)00015-1

Okegawa, Y., Kobayashi, Y., and Shikanai, T. (2010). Physiological links among alternative electron transport pathways that reduce and oxidize plastoquinone in Arabidopsis. Plant J. 63, 458-468. doi: 10.1111/j.1365-313X.2010.04252.x

Ort, D. R., Merchant, S. S., Alric, J., Barkan, A., Blankenship, R. E., Bock, R., et al. (2015). Redesigning photosynthesis to sustainably meet global food and bioenergy demand. Proc. Natl. Acad. Sci. USA 112, 8529-8536. doi: 10.1073/pnas. 1424031112

Park, S., Steen, C. J., Lyska, D., Fischer, A. L., Endelman, B., Iwai, M., et al (2019). Chlorophyll-carotenoid excitation energy transfer and charge transfer in Nannochloropsis oceanica for the regulation of photosynthesis. Proc. Natl. Acad. Sci. USA 116, 3385-3390. doi: 10.1073/pnas.1819011116

Peers, G., Truong, T. B., Ostendorf, E., Busch, A., Elrad, D., Grossman, A. R., et al. (2009). An ancient light-harvesting protein is critical for the regulation of algal photosynthesis. Nature 462, 518-521. doi: 10.1038/nature08587

Petroutsos, D., Tokutsu, R., Maruyama, S., Flori, S., Greiner, A., Magneschi, L., et al. (2016). A blue-light photoreceptor mediates the feedback regulation of photosynthesis. Nature 537, 563-566. doi: 10.1038/nature19358

Pinnola, A., and Bassi, R. (2018). Molecular mechanisms involved in plant photoprotection. Biochem. Soc. Trans. 46, 467-482. doi: 10.1042/BST20170307

Pinnola, A., Dall'Osto, L., Gerotto, C., Morosinotto, T., Bassi, R., and Alboresi, A. (2013). Zeaxanthin binds to light-harvesting complex stress-related protein to enhance nonphotochemical quenching in Physcomitrella patens. Plant Cell 25, 3519-3534. doi: 10.1105/tpc.113.114538

Pribil, M., Sandoval-Ibáñez, O., Xu, W., Sharma, A., Labs, M., Liu, Q., et al. (2018). Fine-tuning of photosynthesis requires curvature thylakoid1-mediated thylakoid plasticity. Plant Physiol. 176, 2351-2364. doi: 10.1104/pp.17.00863

Puthiyaveetil, S., Tsabari, O., Lowry, T., Lenhert, S., Lewis, R. R., Reich, Z., et al. (2014). Compartmentalization of the protein repair machinery in photosynthetic membranes. Proc. Natl. Acad. Sci. USA 111, 15839-15844. doi: 10.1073/pnas.1413739111

Rast, A., Schaffer, M., Albert, S., Wan, W., Pfeffer, S., Beck, F., et al. (2019). Biogenic regions of cyanobacterial thylakoids form contact sites with the plasma membrane. Nat. Plants 5, 436-446. doi: 10.1038/s41477-019-0399-7

Rochaix, J.-D., and Bassi, R. (2019). LHC-like proteins involved in stress responses and biogenesis/repair of the photosynthetic apparatus. Biochem. J. 476, 581-593. doi: 10.1042/BCJ20180718

Ruban, A. V., Berera, R., Ilioaia, C., van Stokkum, I. H. M., Kennis, J. T. M. Pascal, A. A., et al. (2007). Identification of a mechanism of photoprotective energy dissipation in higher plants. Nature 450, 575-578. doi: 10.1038/ nature 06262

Ruban, A. V., Johnson, M. P., Horton, P., Zia, A., and Perez-Bueno, M. L. (2009). The zeaxanthin-independent and zeaxanthin-dependent qE components of nonphotochemical quenching involve common conformational changes within the photosystem II antenna in Arabidopsis. Plant Physiol. 149, 1061-1075. doi: $10.1104 /$ pp.108.129957

Sainz, M., Díaz, P., Monza, J., and Borsani, O. (2010). Heat stress results in loss of chloroplast $\mathrm{Cu} / \mathrm{Zn}$ superoxide dismutase and increased damage to photosystem II in combined drought-heat stressed Lotus japonicus. Physiol. Plant. 140, 46-56. doi: 10.1111/j.1399-3054.2010.01383.x

Salomon, E., Bar-Eyal, L., Sharon, S., and Keren, N. (2013). Balancing photosynthetic electron flow is critical for cyanobacterial acclimation to nitrogen limitation. Biochim. Biophys. Acta Bioenerg. 1827, 340-347. doi: 10.1016/j.bbabio.2012.11.010

Schottkowski, M., Gkalympoudis, S., Tzekova, N., Stelljes, C., Schünemann, D. Ankele, E., et al. (2009). Interaction of the periplasmic pratA factor and the PsbA (D1) protein during biogenesis of photosystem II in synechocystis sp. PCC 6803. J. Biol. Chem. 284, 1813-1819. doi: 10.1074/jbc.M806116200 Sedoud, A., Lopez-Igual, R., Rehman, A. U., Wilson, A., Perreau, F., Boulay, C., et al. (2014). The cyanobacterial photoactive orange carotenoid protein is an excellent singlet oxygen quencher. Plant Cell 26, 1781-1791. doi: 10.1105/ tpc.114.123802 
Silva, P., Thompson, E., Bailey, S., Kruse, O., Mullineaux, C. W., Robinson, C., et al. (2003). FtsH is involved in the early stages of repair of photosystem II in Synechocystis sp. PCC 6803. Plant Cell 15, 2152-2164. doi: 10.1105/tpc.012609

Sirpio, S., Allahverdiyeva, Y., Suorsa, M., Paakkarinen, V., Vainonen, J., Battchikova, N., et al. (2007). TLP18.3, a novel thylakoid lumen protein regulating photosystem II repair cycle. Biochem. J. 406, 415-425. doi: 10.1042/BJ20070460

Slonimskiy, Y. B., Muzzopappa, F., Maksimov, E. G., Wilson, A., Friedrich, T., Kirilovsky, D., et al. (2019). Light-controlled carotenoid transfer between water-soluble proteins related to cyanobacterial photoprotection. FEBS J. 286, 1908-1924. doi: 10.1111/febs.14803

Stengel, A., Gügel, I. L., Hilger, D., Rengstl, B., Jung, H., and Nickelsen, J. (2012). Initial steps of photosystem II de novo assembly and preloading with manganese take place in biogenesis centers in Synechocystis. Plant Cell 24, 660-675. doi: 10.1105/tpc.111.093914

Strzepek, R. F., Boyd, P. W., and Sunda, W. G. (2019). Photosynthetic adaptation to low iron, light, and temperature in Southern Ocean phytoplankton. Proc. Natl. Acad. Sci. USA 116, 4388-4393. doi: 10.1073/pnas.1810886116

Sun, X., Peng, L., Guo, J., Chi, W., Ma, J., Lu, C., et al. (2007). Formation of DEG5 and DEG8 complexes and their involvement in the degradation of photodamaged photosystem II reaction center D1 protein in Arabidopsis. Plant Cell 19, 1347-1361. doi: 10.1105/tpc.106.049510

Takahashi, S., and Badger, M. R. (2011). Photoprotection in plants: a new light on photosystem II damage. Trends Plant Sci. 16, 53-60. doi: 10.1016/j. tplants.2010.10.001

Tian, L., Nawrocki, W. J., Liu, X., Polukhina, I., van Stokkum, I. H. M., and Croce, R. (2019). pH dependence, kinetics and light-harvesting regulation of nonphotochemical quenching in Chlamydomonas. Proc. Natl. Acad. Sci. USA 116, 8320-8325. doi: 10.1073/pnas.1817796116

Tibiletti, T., Auroy, P., Peltier, G., and Caffarri, S. (2016). Chlamydomonas reinhardtii PsbS protein is functional and accumulates rapidly and transiently under high light. Plant Physiol. 171, 2717-2730. doi: 10.1104/pp.16.00572

Tikkanen, M., Nurmi, M., Kangasjärvi, S., and Aro, E.-M. (2008). Core protein phosphorylation facilitates the repair of photodamaged photosystem II at high light. Biochim. Biophys. Acta Bioenerg. 1777, 1432-1437. doi: 10.1016/j. bbabio.2008.08.004

Ting, C. S., and Owens, T. G. (2016). The effects of excess irradiance on photosynthesis in the marine diatom Phaeodactylum tricornutum. Plant Physiol. 106, 763-770. doi: 10.1104/pp.106.2.763

Townsend, A. J., Ware, M. A., and Ruban, A. V. (2018). Dynamic interplay between photodamage and photoprotection in photosystem II. Plant Cell Environ. 41, 1098-1112. doi: 10.1111/pce.13107

Uniacke, J., and Zerges, W. (2007). Photosystem II assembly and repair are differentially localized in Chlamydomonas. Plant Cell 19, 3640-3654. doi: 10.1105/tpc.107.054882

Wagner, D., Przybyla, D., Op Den Camp, R., Kim, C., Landgraf, F., Keun, P. L., et al. (2004). The genetic basis of singlet oxygen-induced stress response of Arabidopsis thaliana. Science 306, 1183-1185. doi: 10.1126/science.1103178
Walker, D. A. (2009). Biofuels, facts, fantasy, and feasibility. J. Appl. Phycol. 21, 509-517. doi: 10.1007/s10811-009-9446-5

Wang, Q., Zhao, H., Jiang, J., Xu, J., Xie, W., Fu, X., et al. (2017). Genetic architecture of natural variation in rice nonphotochemical quenching capacity revealed by genome-wide association study. Front. Plant Sci. 13:1773. doi: $10.3389 /$ fpls.2017.01773

Wei, L., Derrien, B., Gautier, A., Houille-Vernes, L., Boulouis, A., Saint-Marcoux, D., et al. (2014). Nitric oxide-triggered remodeling of chloroplast bioenergetics and thylakoid proteins upon nitrogen starvation in Chlamydomonas reinhardtii. Plant Cell 179, 718-731. doi: 10.1105/tpc.113.120121

Wilk, L., Grunwald, M., Liao, P.-N., Walla, P. J., and Kuhlbrandt, W. (2013). Direct interaction of the major light-harvesting complex II and PsbS in nonphotochemical quenching. Proc. Natl. Acad. Sci. USA 110, 5452-5456. doi: $10.1073 /$ pnas. 1205561110

Wilson, A., Ajlani, G., Verbavatz, J.-M., Vass, I., Kerfeld, C. A., and Kirilovsky, D. (2006). A soluble carotenoid protein involved in phycobilisome-related energy dissipation in cyanobacteria. Plant Cell 18, 992-1007. doi: 10.1105/ tpc. 105.040121

Wilson, S., and Ruban, A. V. (2019). Quantitative assessment of the high-light tolerance in plants with an impaired photosystem II donor side. Biochem. J. 476, 1377-1386. doi: 10.1042/BCJ20190208

Wittenberg, G., Levitan, A., Klein, T., Dangoor, I., Keren, N., and Danon, A. (2014). Knockdown of the Arabidopsis thaliana chloroplast protein disulfide isomerase 6 results in reduced levels of photoinhibition and increased D1 synthesis in high light. Plant J. 78, 1003-1013. doi: 10.1111/tpj.12525

Wobbe, L., Bassi, R., and Kruse, O. (2016). Multi-level light capture control in plants and green algae. Trends Plant Sci. 21, 55-68. doi: 10.1016/j. tplants.2015.10.004

Zhu, X. G., Long, S. P., and Ort, D. R. (2008). What is the maximum efficiency with which photosynthesis can convert solar energy into biomass? Curr. Opin. Biotechnol. 19, 153-159. doi: 10.1016/j.copbio.2008.02.004

Zhu, X., Ort, D. R., Whitmarsh, J., and Long, S. P. (2004). The slow reversibility of photosystem II thermal energy dissipation on transfer from high to low light may cause large losses in carbon gain by crop canopies: a theoretical analysis. J. Exp. Bot. 55, 1167-1175. doi: 10.1093/jxb/erh141

Conflict of Interest Statement: The authors declare that the research was conducted in the absence of any commercial or financial relationships that could be construed as a potential conflict of interest.

Copyright (c) $2019 \mathrm{Liu}, \mathrm{Lu}$, Hua and Last. This is an open-access article distributed under the terms of the Creative Commons Attribution License (CC BY). The use, distribution or reproduction in other forums is permitted, provided the original author(s) and the copyright owner(s) are credited and that the original publication in this journal is cited, in accordance with accepted academic practice. No use, distribution or reproduction is permitted which does not comply with these terms. 